\title{
Research Article \\ Coupling Patterns of External Arguments in the Multiple-Spiral Medallions of the Mandelbrot Set
}

\author{
M. Romera, G. Pastor, A. B. Orue, D. Arroyo, and F. Montoya \\ Instituto de Física Aplicada, CSIC, Serrano 144, 28006 Madrid, Spain \\ Correspondence should be addressed to M. Romera, miguel@iec.csic.es
}

Received 8 March 2009; Revised 2 July 2009; Accepted 16 November 2009

Recommended by B. Sagar

The multiple-spiral medallions are beautiful decorations situated in the proximity of the small copies of the Mandelbrot set. They are composed by an infinity of babies Mandelbrot sets that have external arguments with known structure. In this paper we study the coupling patterns of the external arguments of the baby Mandelbrot sets in multiple-spiral medallions, that is, how these external arguments are grouped in pairs. Based on our experimental data, we obtain that the canonical nonspiral medallions have a nested pairs pattern, the canonical single-spiral medallions have an adjacent pairs pattern, and we conjecture that the canonical double, triple, quadruplespiral medallions have a 1-nested/adjacent pairs pattern.

Copyright (C) 2009 M. Romera et al. This is an open access article distributed under the Creative Commons Attribution License, which permits unrestricted use, distribution, and reproduction in any medium, provided the original work is properly cited.

\section{Introduction}

The Mandelbrot set $M$ was discovered by iterating the complex logistic map $z_{n+1}=\lambda z_{n}\left(1-z_{n}\right)$ [1]. Two years later Douady and Hubbard created the external arguments theory of the $M$ set, starting from the complex map $z_{n+1}=z_{n}^{2}+c$ [2]. The $M$ set can be defined as the set of $c \in \mathrm{C}$ for which the sequence $c, c^{2}+c,\left(c^{2}+c\right)^{2}+c, \ldots$ does not tend to $\infty$. Since the seminal paper of Douady and Hubbard, the $M$ set has been widely studied [3-14], also from a graphical point of view [15-17].

Recently, Wang et al. have studied the generalized $M-J$ sets starting from the complex map $z_{n+1}=z_{n}^{\alpha}+c,(\alpha>1)$. They have proved the connectness of the quaternionic $M$ set [18] and studied the structure and the dynamics of generalized $M-J$ sets $[19,20]$. They also offer a rendering method based on the escape-time to draw the $M$ set [21] and research on the structural characteristic and the fission-evolution law of additive perturbed generalized $M-J$ sets [22]. Finally, they modify the escape-time method and form the preperiod graphic of the generalized $M$ set [23] and also research on the structural characteristic of the generalized $M$ set perturbed by composing noise of additive and multiplicative [24, 25]. 
$M$ contains small copies of itself (babies Mandelbrot sets, BMSs) which in turn contain smaller copies of $M$, and so on ad infinitum. But the $M$ set, as is well known, is not self-similar. Actually, every BMS has its very own pattern of external decorations. There are BMSs with beautiful central symmetry decorations. Some of these decorations are called cauliflowers [26] or embedded Julia sets [27]. Generalizations of the cauliflowers are the multiple-spiral medallions [28].

There is an infinity of BMSs incrusted in the decoration of a multiple-spiral medallion. The external arguments theory of Douady and Hubbard is a valuable tool in order to analyze the Mandelbrot set [29-31]. In this paper, we will study the structure of the multiple-spiral medallions starting from the external arguments of its BMSs. It was conjectured that the pair of binary expansions of the external arguments of the external rays landing at the cusp of the cardioid of a BMS in a multiple-spiral medallion can be written starting from the binary expansions of the external arguments of its "parent" $\left(\overline{p_{1}}, \overline{p_{2}}\right)$ and its "gene" $\left(. \overline{g_{1}}, \overline{g_{2}}\right)$ [28]. Each one of the binary expansions $\left(. \overline{b_{1}}, \overline{b_{2}}\right)$ of the central BMS of a multiple-spiral medallion can be expressed in the form $. \overline{b_{*}}=. \overline{p_{*} p_{*} \cdots p_{*} g_{*}}$, where $\cdot \bar{b}_{*}$ is $\cdot \bar{b}_{1}$ or $\cdot \bar{b}_{2}, \bar{p}_{*}$ is $\cdot \bar{p}_{1}$ or $\cdot \bar{p}_{2}$, and $\cdot \bar{g}_{*}$ is $\bar{g}_{1}$ or. $\bar{g}_{2}$. Each one of the binary expansions $\left(. \overline{\beta_{1}}, \overline{\beta_{2}}\right)$ of a noncentral BMS of a multiple-spiral medallion can be expressed in the form

$$
\overline{\beta_{*}}=\overline{. b_{*} \underbrace{p_{*} p_{*} \cdots p_{*}}_{j}}
$$

where $\cdot \bar{\beta}_{*}$ is. $\bar{\beta}_{1}$ or. $\bar{\beta}_{2}$ and $j$ is the level of the BMS inside the medallion. A given level $j$ has $2^{j}$ BMSs with $2^{j+1}$ different binary expansions.

In this paper, we will study the coupling patterns of the external arguments of the BMSs in a multiple-spiral medallion, that is, how these external arguments are grouped in pairs. We will obtain experimentally, with the computer, the coupling patterns in a lot of (non, single, double, triple ...)-spiral medallions [28], up to the BMSs of the fourth level, by computing the kneading sequences of the external arguments [9]. When this procedure fails, because more than two external arguments have the same kneading sequence, we will draw the medallion with its external rays in order to obtain the coupling pattern [32]. As is known, in this case we could also use the Bruin-Schleicher algorithm [12]. Taking into account the relatively high periods of the BMSs, the recursive Lavaurs's algorithm [6] is not useful in this case.

\section{Coupling Patterns in the Multiple-Spiral Medallions}

\subsection{Symbolic Binary Expansions}

Let us consider the period-4 hyperbolic component located at $-0.1565 \cdots+1.0322 \cdots i$. As is known from Douady and Hubbard [2], this hyperbolic component has the external arguments $(3 / 15,4 / 15)$ in rational form and $(\overline{0011}, \overline{0100})$ in binary expansions form. This hyperbolic component is the parent $\left(\overline{p_{1}}, \overline{p_{2}}\right)=(\overline{0011}, \overline{0100})$ of an infinity of multiple-spiral medallions inserted into its filaments [28]. From here on, we will normally use the binary expansions of the external arguments. The parent is in the wake of the gene $\left(. \overline{g_{1}}, \overline{g_{2}}\right)=$ $(. \overline{001}, \overline{010})$, which is a period-3 disc [28]. In the examples of this paper we, will use this parent and this gene due to their low periods. 
Now, let us consider the period-27 BMS located at $-0.158682143 \cdots+1.039701715 \cdots i$, which is the central BMS of a double-spiral medallion near the above parent. The binary expansions of the external arguments of the external rays landing at the cusp of the cardioid of this central BMS are $\left(\overline{b_{1}}, \overline{b_{2}}\right)=(\overline{001101000011010000110011010}$, $.001101000011010000110100001)$, which can be verified by using the Jung program [15]. Note that we can write, in abbreviated form, $\left(\overline{b_{1}}, \overline{b_{2}}\right)=\left(. \overline{p_{1} p_{2} p_{1} p_{2} p_{1} p_{1} g_{2}}, \overline{p_{1} p_{2} p_{1} p_{2} p_{1} p_{2} g_{1}}\right)=$ $\left(\overline{{\overline{p_{1}}}^{2} p_{1}^{2} g_{2}}, \overline{\overline{p_{1} p_{2}} g_{1}}\right)$ that we name the symbolic binary expansions of the central BMS. In general, as it was conjectured in $[28], \overline{b_{*}}=. \overline{p_{*} p_{*} \cdots p_{*} g_{*}}$.

The former medallion also has notorious noncentral BMSs as, for example, the period-35 one located at $-0.15867218 \cdots+1.03971266 \cdots i$ with binary expansions $\left(\overline{\beta_{1}}, \overline{\beta_{2}}\right)=$ $(\overline{00110100001101000011001101000110100}, \overline{00110100001101000011010000101000011}) \quad$ and symbolic binary expansions $\left(\overline{\beta_{1}}, \overline{\beta_{2}}\right)=\left(\overline{b_{1} p_{1} p_{2}}, \overline{b_{1} p_{2} p_{1}}\right)$, which correspond to a second level BMS. In general, as it was conjectured in [28],

$$
\overline{\beta_{*}}=\overline{. b_{*} \underbrace{p_{*} p_{*} \cdots p_{*}}_{j}} .
$$

\subsection{Final Kneading Sequence}

As is known from [9], the kneading sequence $K(\theta)$ of an external argument $\theta$ is defined as the itinerary of the orbit of $\theta$ under angle doubling, where the itinerary is taken with respect to the partition formed by $\theta / 2$ and $(\theta+1) / 2$. According to [9]

$$
n \text {th entry of } K(\theta)= \begin{cases}0, & \text { if } \frac{(\theta+1)}{2}<2^{n} \theta \bmod 1<\frac{\theta}{2} \\ 1, & \text { if } \frac{\theta}{2}<2^{n} \theta \bmod 1<\frac{(\theta+1)}{2} \\ 1 & , \quad \text { if } 2^{n} \theta \bmod 1=\frac{\theta}{2} \\ 0 & \text { if } 2^{n} \theta \bmod 1=\frac{(\theta+1)}{2} .\end{cases}
$$

Let $\left(\theta_{1}, \theta_{2}\right)$ be the external arguments of a hyperbolic component, where $\theta_{1}<\theta_{2}$. It is known that $K\left(\theta_{1}\right)=K\left(\theta_{2}\right)$ with the exception of the last digits ${ }_{0}^{1}$ and ${ }_{1}^{0}$. For instance, when $\left(\theta_{1}, \theta_{2}\right)=(3 / 15,4 / 15)$, external arguments of a period-4 hyperbolic component, we obtain the kneading sequences $K\left(\theta_{1}\right)=\overline{110_{1}^{0}}$ and $K\left(\theta_{2}\right)=\overline{110_{0}^{1}}$, where the three first digits are the same.

Let us consider a multiple-spiral medallion, and let $\left(. \overline{b_{1}}, \overline{b_{2}}\right)$ be the binary expansions of its period- $n$ central BMS. The first $n-1$ digits of the kneading sequences of.$\overline{b_{1}}$ and.$\overline{b_{2}}$ are the same. Let

$$
\left(\overline{\overline{b_{*} p_{*} p_{*} \cdots p_{*}}}, \overline{b_{j}^{b_{*} p_{*} p_{*} \cdots p_{*}}}\right)
$$




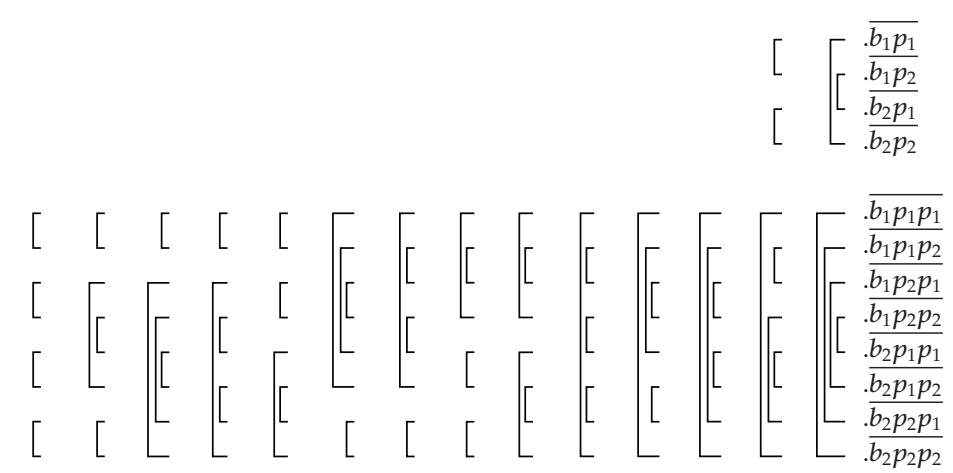

Figure 1: Possible coupling patterns in the first and second levels $\overline{. b_{*} p_{*}}$ and.$\overline{b_{*} p_{*} p_{*}}$ corresponding to the noncentral BMSs in a multiple-spiral medallion.

be the symbolic binary expansions of a noncentral BMS of level $j$ of the same medallion. It is evident that the first $n-1$ digits of the kneading sequences of the external arguments of the noncentral BMS are the same. To compare the kneading sequences corresponding to the BMSs of a given level in a medallion, it is not necessary to handle all the digits of the kneading sequences because the first $n-1$ digits are the same. In this paper, we will use the final kneading sequences, that is, the kneading sequences without the first $n-1$ digits, where $n$ is the period of the central BMS of the medallion.

\subsection{The Coupling Problem}

We name a multiple-spiral medallion by writing the pair of symbolic binary expansions $\left(\overline{b_{1}}, \overline{b_{2}}\right)$ corresponding to its central BMS. The coupling problem consists in knowing how the external arguments are coupled in pairs in each one of the noncentral BMSs of a multiplespiral medallion.

Let $\left(. \overline{p_{1}}, \overline{p_{2}}\right)$ be the binary expansions of the parent of the medallion and let $\overline{b_{1} p_{1}}<$ $\overline{b_{1} p_{2}}<\overline{b_{2} p_{1}}<\overline{. b_{2} p_{2}}$ be the four symbolic binary expansions of the first level BMSs. We can group them in couples in the forms $\left\{\left(\overline{b_{1} p_{1}}, \overline{b_{2} p_{2}}\right),\left(\overline{b_{1} p_{2}}, \overline{b_{2} p_{1}}\right)\right\}$ and $\left\{\left(\overline{b_{1} p_{1}}, \overline{b_{1} p_{2}}\right)\right.$, $\left.\left(\overline{b_{2} p_{1}}, \overline{b_{2} p_{2}}\right)\right\}$ (see Figure 1$)$. Note that the grouping $\left\{\left(\overline{. b_{1} p_{1}}, \overline{b_{2} p_{1}}\right),\left(\overline{b_{1} p_{2}}, \overline{b_{2} p_{2}}\right)\right\}$ is not possible because the corresponding external rays intersect. Therefore, in the first level we have $N_{1}=2$ coupling patterns.

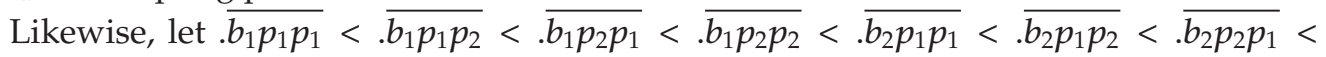
$\overline{b_{2} p_{2} p_{2}}$ be the eight symbolic binary expansions of the second level BMSs of a medallion. In Figure 1 , we show the resulting $N_{2}=14$ coupling patterns by joining the binary expansions of each couple with a straight line.

The number of possible coupling patterns in a given level increases very rapidly with the level. For instance, in the third level we have counted $N_{3}=1374$ coupling patterns, and $N_{l} \geq N_{l-1}+\sum_{2^{l-1}+1}^{2^{l}} 3^{i-2}$ is a low limit of the number of coupling patterns corresponding to the level $l$.

However, in each level three patterns with a strong symmetry can be found, which we will call the canonical coupling patterns: the nested pairs pattern, the adjacent pairs pattern, 
Table 1: Location of canonical nonspiral medallions.

\begin{tabular}{ll}
\hline$\left(\overline{p_{1} p_{2}^{i} g_{2}}, \overline{p_{2} p_{1}^{i} g_{1}}\right)$ & Location \\
\hline$i=1$ & $-0.172595 \cdots+1.050337 \cdots i$ \\
$i=2$ & $-0.1703025 \cdots+1.0445545 \cdots i$ \\
$i=3$ & $-0.16941127 \cdots+1.04295087 \cdots i$ \\
$i=11$ & $-0.16898713353 \cdots+1.04237025723 \cdots i$ \\
\hline
\end{tabular}

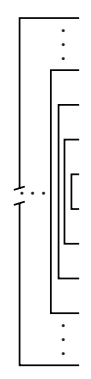

(a)

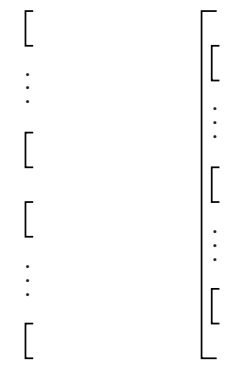

(b)

$$
\left[\begin{array}{l}
{[} \\
\vdots \\
{[} \\
\vdots \\
{[}
\end{array}\right.
$$

(c)

Figure 2: Canonical coupling patterns. (a) Nested pairs pattern. (b) Adjacent pairs pattern. (c) 1-nested/adjacent pairs pattern.

and the 1-nested/adjacent pairs pattern (Figure 2). In the following paragraphs, we will locate multiple-spiral medallions with canonical coupling patterns and we will give examples with both the symbolic binary expansions and the complex coordinates of their central BMSs.

\section{Coupling Pattern of Canonical Nonspiral Medallions}

Let $\left(\overline{p_{1}}, \overline{p_{2}}\right)$ and $\left(\overline{g_{1}}, \overline{g_{2}}\right)$ be the parent and the gene of a nonspiral medallion. As is known from $[33,34]$, the order- $i$ harmonic of the parent located at its main antenna is characterized by the symbolic binary expansions $\left(\overline{p_{1} p_{2}^{i}}, \overline{p_{2} p_{1}^{i}}\right)$, and the limit when $i \rightarrow \infty$ is the tip of the main antenna, which is the Misiurewicz point with preperiodic symbolic binary expansions $\left(. p_{1} \overline{p_{2}}, p_{2} \overline{p_{1}}\right)$. As is also known, the nonspiral medallions are located near the tip and outside of the parent [28]. We name canonical nonspiral medallions those whose central BMSs have the symbolic binary expansions $\left(\overline{p_{1} p_{2}^{i} g_{2}}, \overline{p_{2} p_{1}^{i} g_{1}}\right)$ with $i=1,2 \ldots$ The limit of the nonspiral medallions when $i \rightarrow \infty$ is the tip of the parent. In Figure 3(b), we can observe the locations of the $i=1,2,3,11$ canonical nonspiral medallions corresponding to the parent and the gene of Figure 3(a). The locations of the noncentral BMSs of the nonspiral medallion $i=11$, up to third level, can be seen in Figure 3(c).

Obviously, a nonspiral medallion has no spiral. However, we include them in the family of multiple-spiral medallions for two reasons. First, because they are located in the filaments of the parent, as the rest of the multiple-spiral medallions; and second, because the symbolic binary expansions of their central BMSs can be obtained in the same manner as the rest of the medallions (see [28, Figure 8]).

In Table 1 the complex coordinates of some canonical nonspiral medallions are given. 


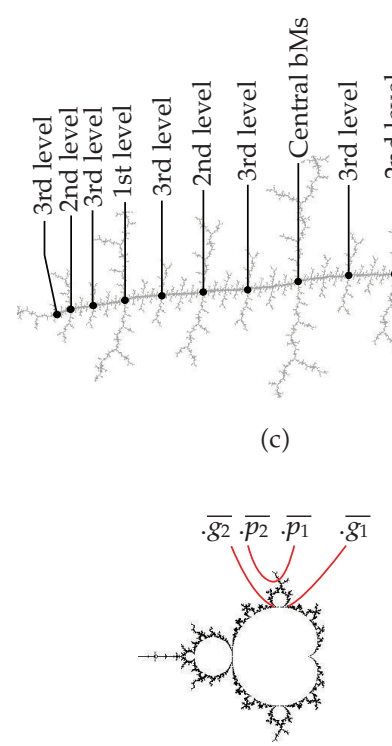

(a)

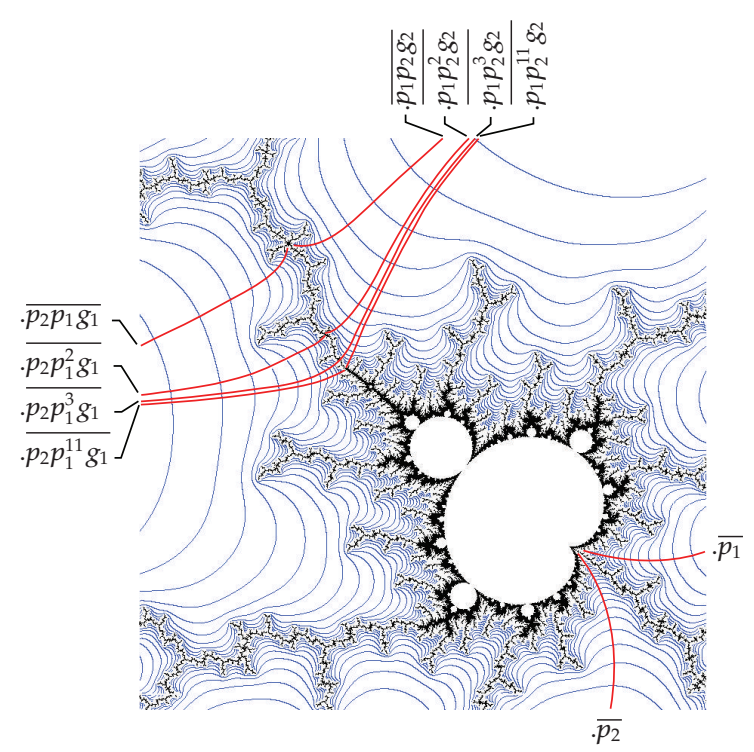

(b)

Figure 3: Canonical nonspiral medallions. (a) External rays of the parent and the gene. (b) Graphical location of the medallions $i=1,2,3$ and 11. (c) Detail of the medallion $i=11$, where the BMSs up to third level are pointed out.

We have experimentally obtained the coupling patterns of the canonical nonspiral medallions of Table 1, starting from the final kneading sequences up to fourth level BMSs. All of these coupling patterns are the nested pairs pattern of Figure 4.

\section{Coupling Pattern of Canonical Single-Spiral Medallions}

The single-spiral medallions are known as "cauliflowers" $[14,26,33]$. Let $\left(. \overline{p_{1}}, \overline{p_{2}}\right)$ and $\left(\overline{g_{1}}, \overline{g_{2}}\right)$ be the parent and the gene of a single-spiral medallion. As is known from $[33,34]$, the order- $(i-1)$ antiharmonic of the parent has the binary expansions $\left(. p_{1}^{i}, \overline{p_{2}^{i}}\right)$. We will call canonical single-spiral medallions to those whose central BMSs have the symbolic binary expansions $\left(. \overline{p_{1}^{i} g_{1}}, \overline{p_{2}^{i} g_{2}}\right)$ with $i=1,2 \ldots$ These medallions are inserted in the filament of the cusp of the parent, and its limit when $i=\infty$ is this cusp. In Figure 5(b), we can see the locations of the $i=1,2,3$ canonical single-spiral medallions corresponding to the parent and the gene of Figure 5(a). The locations of the noncentral BMSs in the single-spiral medallion $i=14$, up to third level, are shown in Figure 5(c).

In Table 2, the complex coordinates of some canonical single-spiral medallions are given.

The coupling pattern of a single-spiral medallion cannot be obtained by using the final kneading sequences since the kneading sequences of more than two of its noncentral BMSs are the same. However, we have two new options. First, we can use the Bruin and Schleicher algorithm to find conjugate external arguments [12]. Second, we can draw the single-spiral medallion with its external rays (in each level $j$, we have $2^{j+1}$ external rays and $2^{j+1}$ symbolic binary expansions) and later to assign the correct symbolic binary expansion to 
Table 2: Location of canonical single-spiral medallions.

\begin{tabular}{ll}
\hline$\left(\overline{p_{1}^{i} g_{1}}, \overline{p_{2}^{i} g_{2}}\right)$ & Location \\
\hline$i=1$ & $-0.12749 \cdots+0.98746 \cdots i$ \\
$i=2$ & $-0.143322 \cdots+1.018962 \cdots i$ \\
$i=3$ & $-0.1488893 \cdots+1.0259091 \cdots i$ \\
$i=8$ & $-0.15375614 \cdots+1.03038322 \cdots i$ \\
$i=14$ & $-0.1543869391 \cdots+1.0308295094 \cdots i$ \\
\hline
\end{tabular}

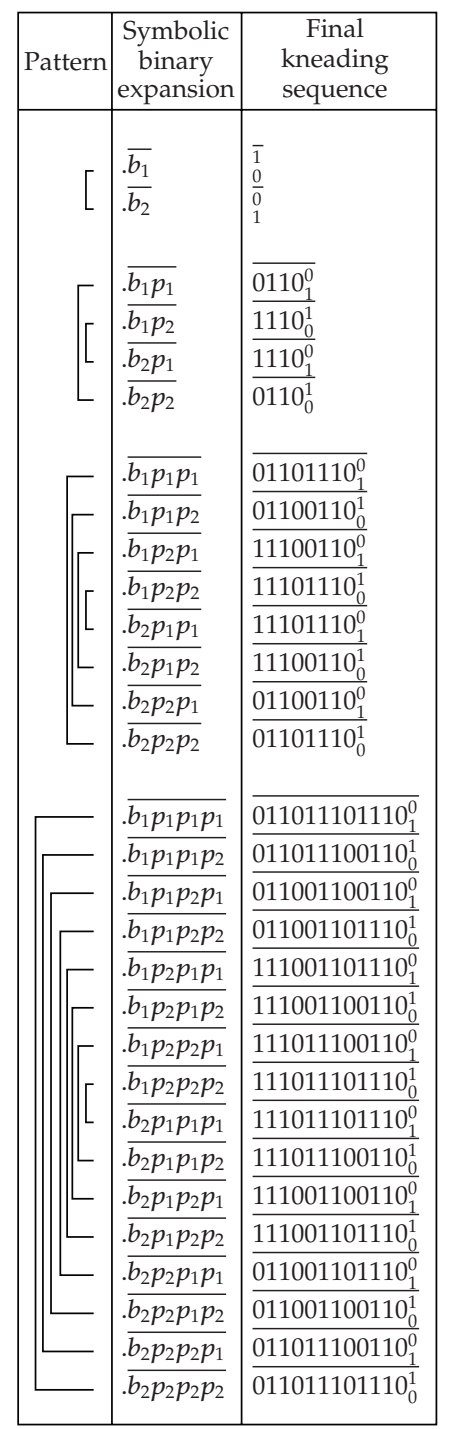

\begin{tabular}{|c|c|c|}
\hline Pattern & $\begin{array}{c}\text { Symbolic } \\
\text { binary } \\
\text { expansion }\end{array}$ & $\begin{array}{c}\text { Final } \\
\text { kneading } \\
\text { sequence }\end{array}$ \\
\hline &.$b_{1} p_{1} p_{1} p_{1} p_{1}$ & $0110111011101110^{0}$ \\
\hline &.$\overline{b_{1} p_{1} p_{1} p_{1} p_{2}}$ & $\overline{0110111011100110_{0}^{1}}$ \\
\hline & $\overline{b_{1} p_{1} p_{1} p_{2} p_{1}}$ & $0110111001100110_{1}^{0}$ \\
\hline & $\overline{b_{1} p_{1} p_{1} p_{2} p_{2}}$ & $0110111001101110_{0}^{1}$ \\
\hline &. $\bar{b}_{1} p_{1} p_{2} p_{1} p_{1}$ & $0110011001101110_{1}^{0}$ \\
\hline &.$b_{1} p_{1} p_{2} p_{1} p_{2}$ & $\underline{0110011001100110_{0}^{1}}$ \\
\hline & $\overline{b_{1} p_{1} p_{2} p_{2} p_{1}}$ & $0110011011100110_{1}^{0}$ \\
\hline &.$\overline{b_{1} p_{1} p_{2} p_{2} p_{2}}$ & $0110011011101110_{0}^{1}$ \\
\hline &.$\overline{b_{1} p_{2} p_{1} p_{1} p_{1}}$ & $1110011011101110_{1}^{0}$ \\
\hline &.$\overline{b_{1} p_{2} p_{1} p_{1} p_{2}}$ & $1110011011100110_{0}^{1}$ \\
\hline &.$\overline{b_{1} p_{2} p_{1} p_{2} p_{1}}$ & $1110011001100110_{1}^{0}$ \\
\hline &.$b_{1} p_{2} p_{1} p_{2} p_{2}$ & $1110011001101110_{0}^{1}$ \\
\hline &.$b_{1} p_{2} p_{2} p_{1} p_{1}$ & $1110111001101110_{1}^{0}$ \\
\hline & $b_{1} p_{2} p_{2} p_{1} p_{2}$ & $1110111001100110_{0}^{1}$ \\
\hline &.$b_{1} p_{2} p_{2} p_{2} p_{1}$ & $1110111011100110_{1}^{0}$ \\
\hline &.$b_{1} p_{2} p_{2} p_{2} p_{2}$ & $1110111011101110_{0}^{1}$ \\
\hline &.$b_{2} p_{1} p_{1} p_{1} p_{1}$ & $1110111011101110_{1}^{0}$ \\
\hline &.$b_{2} p_{1} p_{1} p_{1} p_{2}$ & $1110111011100110_{0}^{1}$ \\
\hline &.$b_{2} p_{1} p_{1} p_{2} p_{1}$ & $1110111001100110_{1}^{0}$ \\
\hline &.$b_{2} p_{1} p_{1} p_{2} p_{2}$ & $1110111001101110_{0}^{1}$ \\
\hline &.$b_{2} p_{1} p_{2} p_{1} p_{1}$ & $\overline{1110011001101110_{1}^{0}}$ \\
\hline &.$b_{2} p_{1} p_{2} p_{1} p_{2}$ & $1110011001100110_{0}^{1}$ \\
\hline &.$b_{2} p_{1} p_{2} p_{2} p_{1}$ & $1110011011100110_{1}^{0}$ \\
\hline &.$\overline{b_{2} p_{1} p_{2} p_{2} p_{2}}$ & $1110011011101110_{0}^{1}$ \\
\hline &.$\overline{b_{2} p_{2} p_{1} p_{1} p_{1}}$ & $0110011011101110_{1}^{0}$ \\
\hline &.$b_{2} p_{2} p_{1} p_{1} p_{2}$ & $0110011011100110_{0}^{1}$ \\
\hline &.$\overline{b_{2} p_{2} p_{1} p_{2} p_{1}}$ & $0110011001100110_{1}^{0}$ \\
\hline & $\bar{b}_{2} p_{2} p_{1} p_{2} p_{2}$ & $\underline{0110011001101110_{0}^{1}}$ \\
\hline &.$b_{2} p_{2} p_{2} p_{1} p_{1}$ & $0110111001101110_{1}^{0}$ \\
\hline &.$\overline{b_{2} p_{2} p_{2} p_{1} p_{2}}$ & $\underline{0110111001100110_{0}^{1}}$ \\
\hline &.$b_{2} p_{2} p_{2} p_{2} p_{1}$ & $0110111011100110_{1}^{0}$ \\
\hline & $b_{2} p_{2} p_{2} p_{2} p_{2}$ & $0110111011101110_{0}^{1}$ \\
\hline
\end{tabular}

Figure 4: Coupling pattern of the external arguments of the BMSs up to fourth level in a canonical nonspiral medallion. The corresponding final kneading sequences are shown. 


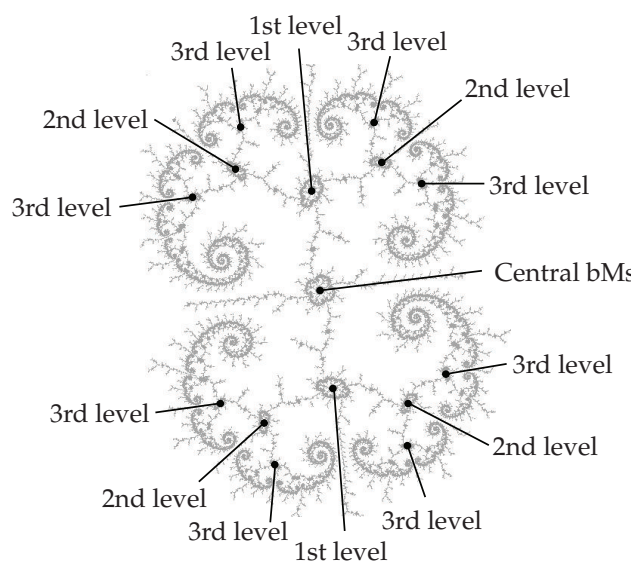

(c)

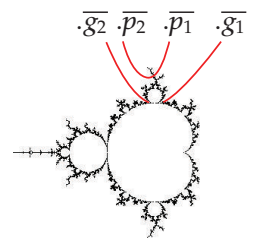

(a)

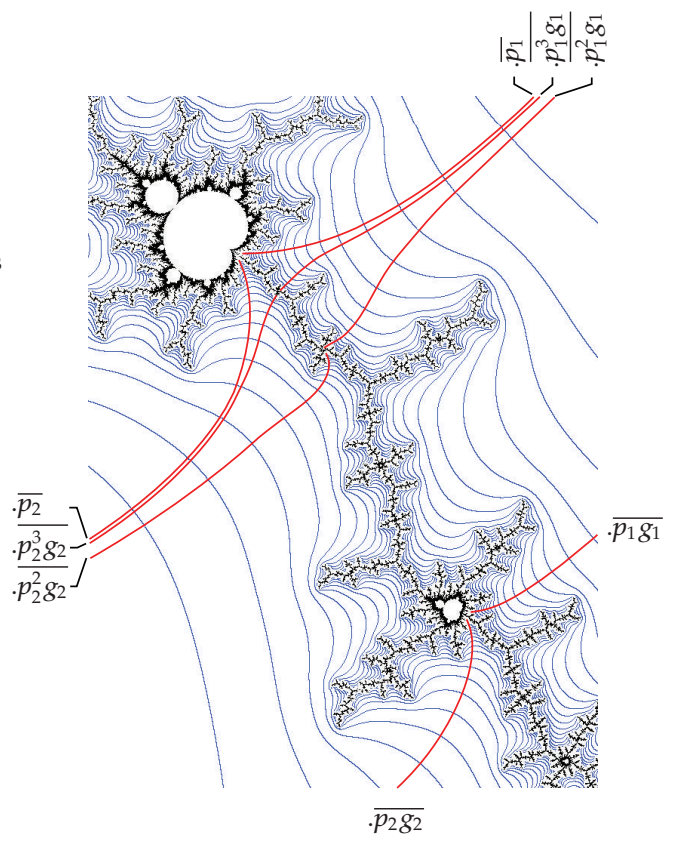

(b)

Figure 5: Canonical single-spiral medallions. (a) External rays of the parent and the gene. (b) Graphical location of the medallions $i=1,2$ and 3. (c) Detail of the medallion $i=14$ where the BMSs up to third level are pointed out.

each external ray by simple ordering [32]. We have chosen the last option since the drawing of the $i=8$ canonical single-spiral medallion was already performed in a previous paper (see [32, Figure 7]). Starting from this figure, the coupling pattern of a canonical single-spiral medallion corresponds to the adjacent pairs pattern of Figure 6.

\section{Coupling Pattern of Canonical Double-Spiral Medallions}

Let us consider the gene $\left(. \overline{g_{1}}, \overline{g_{2}}\right)$ and the period- $n$ parent $\left(. \overline{p_{1}}, \overline{p_{2}}\right)$ of a double-spiral medallion. As is known from the tuning algorithm [3], the external rays landing at the tangent point of the cardioid of the parent with its period- $2 n$ disc have the symbolic binary expansions $\left(\overline{p_{1} p_{2}}, \overline{p_{2} p_{1}}\right)$. The double-spiral medallions are inserted in the filaments near these external rays, and the structures.$\overline{p_{1} p_{2}}$ and.$\overline{p_{2} p_{1}}$ appear in the symbolic binary expansions of the central BMS of a double-spiral medallion. We name canonical double-spiral medallions

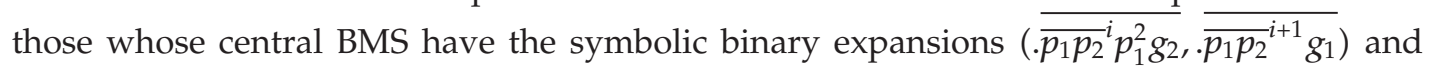
$\left(\overline{\overline{p_{2} p_{1}}}{ }^{i+1} g_{2}, \overline{\overline{p_{2} p_{1}}} p_{2}^{2} g_{1}\right)$. In Figure $7(\mathrm{~b})$ we can see the locations of the $i=1,2$ canonical doublespiral medallions corresponding to the parent and the gene of Figure 7(a). The locations of the noncentral BMSs in the double-spiral medallion $\left(\overline{\overline{p_{1} p_{2}}}{ }^{16} p_{1}^{2} g_{2}, \overline{{\overline{p_{1} p_{2}}}^{17} g_{1}}\right)$, up to third level, are shown in Figure 7(c). In Table 3 the complex coordinates of some canonical double-spiral medallions are given. 


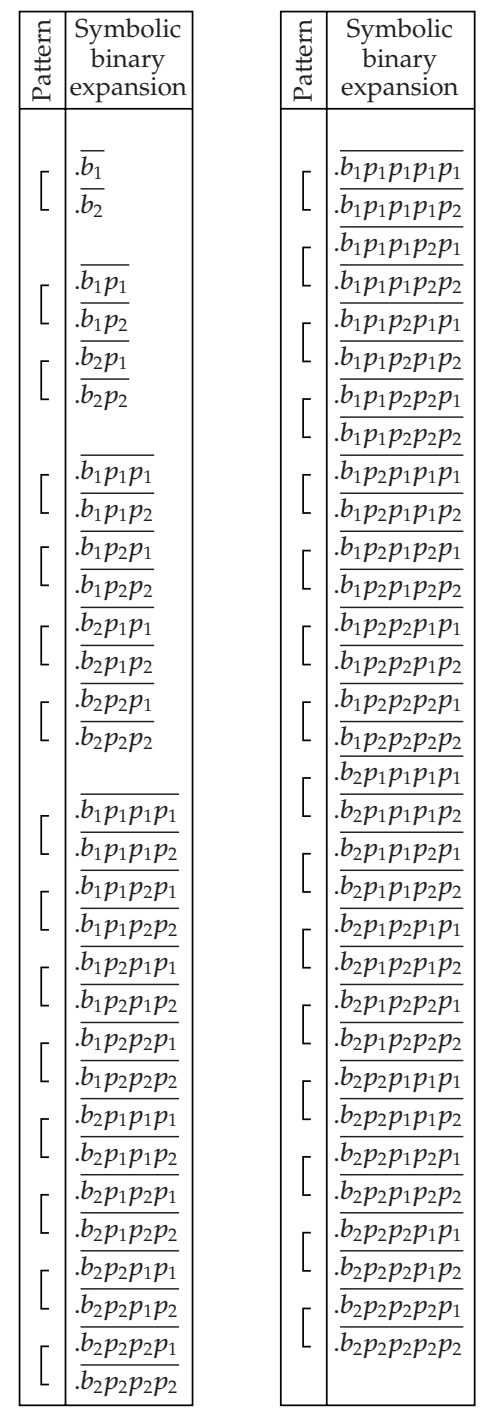

Figure 6: Coupling pattern of the external arguments of the BMSs up to fourth level in a canonical singlespiral medallion.

The coupling pattern of the canonical double-spiral medallions can be obtained from the final kneading sequences. The result is the 1-nested/adjacent pairs pattern which can be seen in Figure 8.

\section{Coupling Pattern of Canonical High-Order-Spiral Medallions}

Let us consider the gene $\left(. \overline{g_{1}}, \overline{g_{2}}\right)$ and the period- $n$ parent $\left(. \overline{p_{1}}, \overline{p_{2}}\right)$ of a triple-spiral medallion. As is known from the tuning algorithm [3], the external rays landing at the tangent point of the cardioid of the parent with its period- $3 n$ discs have the binary expansions $\left(. \overline{p_{1}^{2} p_{2}}, \overline{p_{1} p_{2} p_{1}}\right)$ and $\left(. \overline{p_{2} p_{1} p_{2}}, \overline{p_{2}^{2} p_{1}}\right)$. These structures appear in the symbolic binary expansions of the central 
Table 3: Location of canonical double-spiral medallions.

\begin{tabular}{|c|c|}
\hline$\left({\overline{\bar{p}_{1} p_{2}}}^{i} p_{1}^{2} g_{2}, \cdot \overline{{\overline{p_{1} p_{2}}}^{i+1} g_{1}}\right)$ & Location \\
\hline$\overline{i=1}$ & $-0.1566489 \cdots+1.0417901 \cdots i$ \\
\hline$i=2$ & $-0.15868214 \cdots+1.03970171 \cdots i$ \\
\hline$i=3$ & $-0.159450890 \cdots+1.038682639 \cdots i$ \\
\hline$i=16$ & $-0.16090253699 \cdots+1.03660106997 \cdots i$ \\
\hline
\end{tabular}

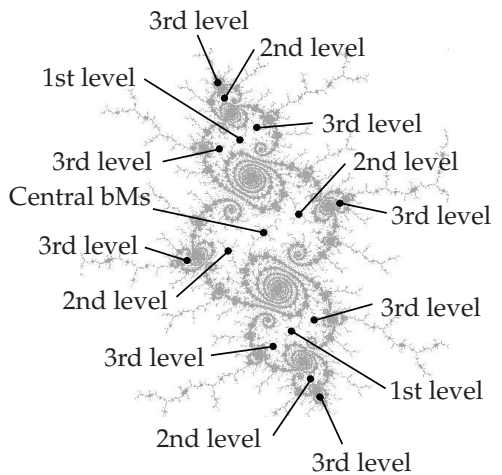

(c)

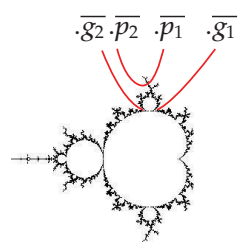

(a)

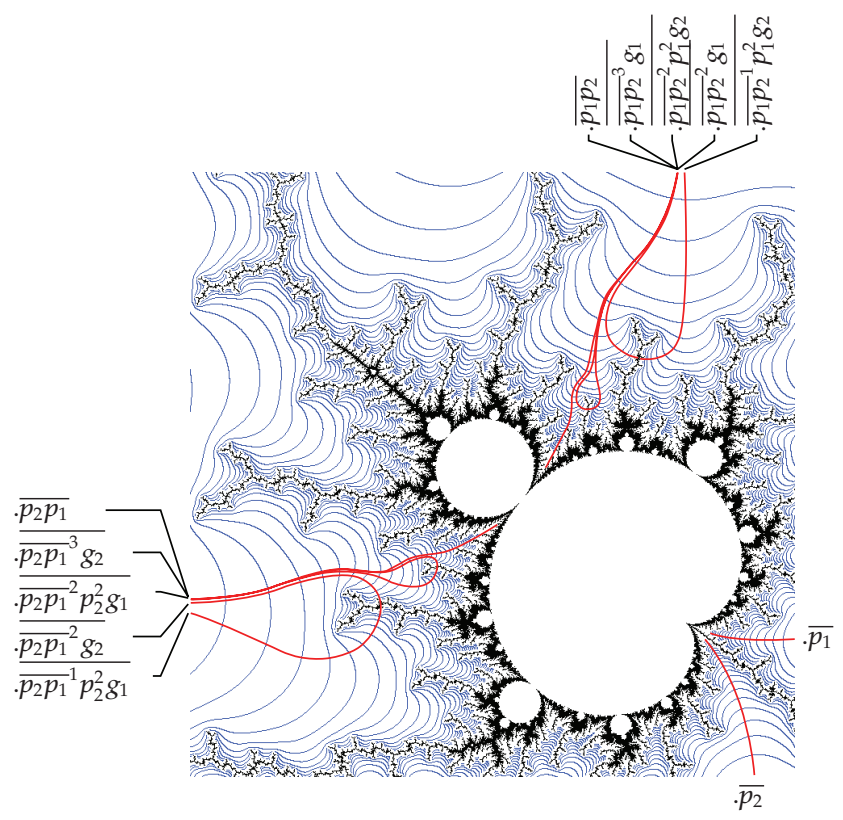

(b)

Figure 7: Canonical double-spiral medallions. (a) External rays of the parent and the gene. (b) Graphical location of the medallions $i=1$ and 2. (c) Detail of the medallion $\left(\overline{{\overline{p_{1} p_{2}}}^{16} p_{1}^{2} g_{2}}, \overline{{\overline{p_{1} p_{2}}}^{17} g_{1}}\right)$ where the BMSs up to third level are pointed out.

BMS of a triple-spiral medallion. In Figure 9(b) we can observe the locations of the $i=$

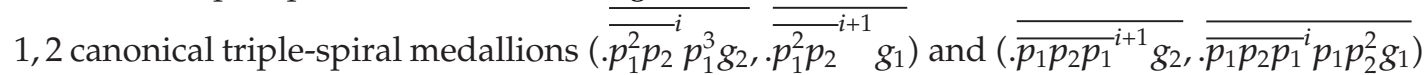
corresponding to the parent and the gene of Figure $9(a)$. The situations of the noncentral BMSs in the canonical triple-spiral medallion $\left(. \overline{\bar{p}_{1}^{2} p_{2}} p_{1}^{11} g_{2}, \overline{{\overline{p_{1}^{2} p_{2}}}^{12} g_{1}}\right)$, up to third level, are pointed out in Figure 9(c). In Table 4 the complex coordinates of some canonical triple-spiral medallions are given.

The coupling pattern of the canonical triple-spiral medallions can be obtained from the final kneading sequences. The result is again the 1-nested/adjacent pairs pattern which can be seen in Figure 8, as in the case of the double-spiral medallions.

We have found canonical high order-spiral medallions in order to see their coupling patterns. In Tables 5, 6, and 7 the complex coordinates of some of these medallions are given.

The coupling patterns of canonical quadruple, quintuple and 20.tuple-spiral medallions correspond once again to the 1-nested/adjacent pairs pattern of Figure 8, similarly to 


\begin{tabular}{|c|c|c|c|}
\hline$\underbrace{\circ}_{\sigma}$ & $\begin{array}{c}\text { Symbolic } \\
\text { binary } \\
\text { expansion }\end{array}$ & 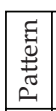 & $\begin{array}{c}\text { Symbolic } \\
\text { binary } \\
\text { expansion }\end{array}$ \\
\hline & $\overline{b_{1}}$ & Г &.$\overline{b_{1} p_{1} p_{1} p_{1} p_{1}}$ \\
\hline &.$\overline{b_{2}}$ & &. \\
\hline & & L & $\overline{. \overline{b_{1} p_{1} p_{1} p_{2} p_{1}}}$ \\
\hline$\Gamma$ & $\overline{b_{1} p_{1}}$ & г &.$\overline{b_{1} p_{1} p_{1} p_{2} p_{2}}$ \\
\hline & $\overline{b_{1} p_{2}}$ & 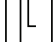 & $\overline{. b_{1} p_{1} p_{2} p_{1} p_{1}}$ \\
\hline & $\overline{b_{2} p_{1}}$ & ] &.$\overline{b_{1} p_{1} p_{2} p_{1} p_{2}}$ \\
\hline L & $\overline{b_{2} p_{2}}$ & L & $\overline{\overline{b_{1} p_{1} p_{2} p_{2} p_{1}}}$ \\
\hline & & &.$\overline{b_{1} p_{1} p_{2} p_{2} p_{2}}$ \\
\hline T &.$\overline{b_{1} p_{1} p_{1}}$ & L &.$\overline{b_{1} p_{2} p_{1} p_{1} p_{1}}$ \\
\hline & $\overline{b_{1} p_{1} p_{2}}$ & &.$\overline{b_{1} p_{2} p_{1} p_{1} p_{2}}$ \\
\hline & $\overline{b_{1} p_{2} p_{1}}$ & L & $\overline{b_{1} p_{2} p_{1} p_{2} p_{1}}$ \\
\hline & $\overline{b_{1} p_{2} p_{2}}$ & & $\overline{b_{1} p_{2} p_{1} p_{2} p_{2}}$ \\
\hline & $\overline{b_{2} p_{1} p_{1}}$ & IL & $\overline{b_{1} p_{2} p_{2} p_{1} p_{1}}$ \\
\hline & $\overline{b_{2} p_{1} p_{2}}$ & &.$\overline{b_{1} p_{2} p_{2} p_{1} p_{2}}$ \\
\hline & $\overline{b_{2} p_{2} p_{1}}$ & L & $\overline{b_{1} p_{2} p_{2} p_{2} p_{1}}$ \\
\hline L &.$\overline{b_{2} p_{2} p_{2}}$ & 斤 &.$\overline{b_{1} p_{2} p_{2} p_{2} p_{2}}$ \\
\hline & & L & $\frac{b_{2} p_{1} p_{1} p_{1} p_{1}}{b_{2} p_{1} p_{1} p_{1} p^{\prime}}$ \\
\hline & $\overline{b_{1} p_{1} p_{1} p_{1}}$ & [ &.$\overline{b_{2} p_{1} p_{1} p_{1} p_{2}}$ \\
\hline & $\overline{b_{1} p_{1} p_{1} p_{2}}$ & L & $\overline{b_{2} p_{1} p_{1} p_{2} p_{1}}$ \\
\hline & b & &.$\overline{b_{2} p_{1} p_{1} p_{2} p_{2}}$ \\
\hline & $\overline{b_{1} p_{1} p_{2} p_{2}}$ & L &.$\overline{b_{2} p_{1} p_{2} p_{1} p_{1}}$ \\
\hline &.$\overline{b_{1} p_{2} p_{1} p_{1}}$ & I &.$\overline{b_{2} p_{1} p_{2} p_{1} p_{2}}$ \\
\hline & $\overline{b_{1} p_{2} p_{1} p_{2}}$ & L &.$\overline{b_{2} p_{1} p_{2} p_{2} p_{1}}$ \\
\hline & $\overline{b_{1} p_{2} p_{2} p_{1}}$ & &.$\overline{b_{2} p_{1} p_{2} p_{2} p_{2}}$ \\
\hline & $\overline{b_{1} p_{2} p_{2} p_{2}}$ & L &.$\overline{b_{2} p_{2} p_{1} p_{1} p_{1}}$ \\
\hline & $\overline{b_{2} p_{1} p_{1} p_{1}}$ & |Г &.$\overline{b_{2} p_{2} p_{1} p_{1} p_{2}}$ \\
\hline & $\overline{b_{2} p_{1} p_{1} p_{2}}$ & L & $\overline{b_{2} p_{2} p_{1} p_{2} p_{1}}$ \\
\hline & $\overline{b_{2} p_{1} p_{2} p_{1}}$ & | &.$\overline{b_{2} p_{2} p_{1} p_{2} p_{2}}$ \\
\hline & $\overline{b_{2} p_{1} p_{2} p_{2}}$ & L & $\overline{b_{2} p_{2} p_{2} p_{1} p_{1}}$ \\
\hline & $\overline{b_{2} p_{2} p_{1} p_{1}}$ & $\mid \Gamma$ &.$\overline{b_{2} p_{2} p_{2} p_{1} p_{2}}$ \\
\hline & $\overline{b_{2} p_{2} p_{1} p_{2}}$ & L &.$\overline{b_{2} p_{2} p_{2} p_{2} p_{1}}$ \\
\hline & $\overline{b_{2} p_{2} p_{2} p_{1}}$ & L & $\overline{b_{2} p_{2} p_{2} p_{2} p_{2}}$ \\
\hline & $\overline{b_{2} p_{2} p_{2} p_{2}}$ & & \\
\hline
\end{tabular}

Figure 8: Coupling pattern of the external arguments of the BMSs up to fourth level in a canonical doublespiral medallion.

the cases of the double and triple-spiral medallions. Therefore, we can conjecture that the canonical $n$.tuple-spiral medallions $(n \geq 2)$ have a 1-nested/adjacent pairs pattern.

\section{Conclusions}

The coupling patterns of the canonical multiple-spiral medallions have been experimentally studied. Two experimental methods have been used to find the coupling patterns: the first one by using the kneading sequences, and the second one by using the handmade drawing of external rays and the later ordering of their external arguments. 
Table 4: Location of canonical triple-spiral medallions.

\begin{tabular}{|c|c|}
\hline 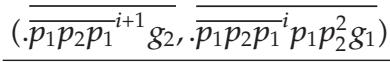 & Location \\
\hline$i=1$ & $-0.1562143 \cdots+1.0397508 \cdots i$ \\
\hline$i=2$ & $-0.15553568 \cdots+1.03854489 \cdots i$ \\
\hline$i=3$ & $-0.155244096 \cdots+1.038069528 \cdots i$ \\
\hline$i=11$ & $-0.1546766523 \cdots+1.0373581018 \cdots i$ \\
\hline
\end{tabular}

Table 5: Location of canonical quadruple-spiral medallions.

\begin{tabular}{ll}
\hline$\left.\overline{\overline{\bar{p}_{1}^{2} p_{2} p_{1}}}{ }^{i+1} g_{2}, \overline{{\overline{p_{1}^{2} p_{2} p_{1}}}^{i} p_{1}^{2} p_{2}^{2} g_{1}}\right)$ & Location \\
\hline$i=1$ & $-0.152235840 \cdots+1.036053596 \cdots i$ \\
$i=2$ & $-0.1524782284 \cdots+1.0354175710 \cdots i$ \\
$i=3$ & $-0.15255235393 \cdots+1.03515698018 \cdots i$ \\
$i=8$ & $-0.152602024868 \cdots+1.034789268092 \cdots i$ \\
\hline
\end{tabular}

Table 6: Location of canonical quintuple-spiral medallions.

\begin{tabular}{|c|c|}
\hline$\left.\overline{\left(\overline{p_{1}^{3} p_{2} p_{1}}\right.}{ }^{i+1} g_{2}, \overline{\bar{p}_{1}^{3} p_{2} p_{1}}{ }^{i} p_{1}^{3} p_{2}^{2} g_{1}\right)$ & Location \\
\hline$i=1$ & $-0.15200993 \cdots+1.03357269 \cdots i$ \\
\hline$i=2$ & $-0.152295958 \cdots+1.033337933 \cdots i$ \\
\hline$i=3$ & $-0.1523987430 \cdots+1.0332321714 \cdots i$ \\
\hline$i=6$ & $-0.152493713878 \cdots+1.033103509887 \cdots i$ \\
\hline
\end{tabular}

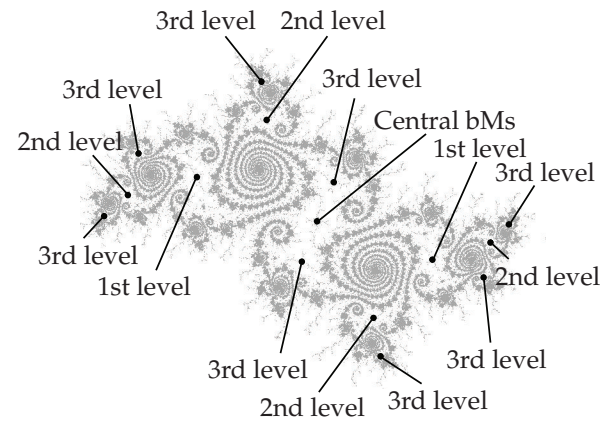

(c)

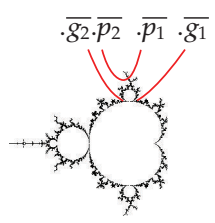

(a)

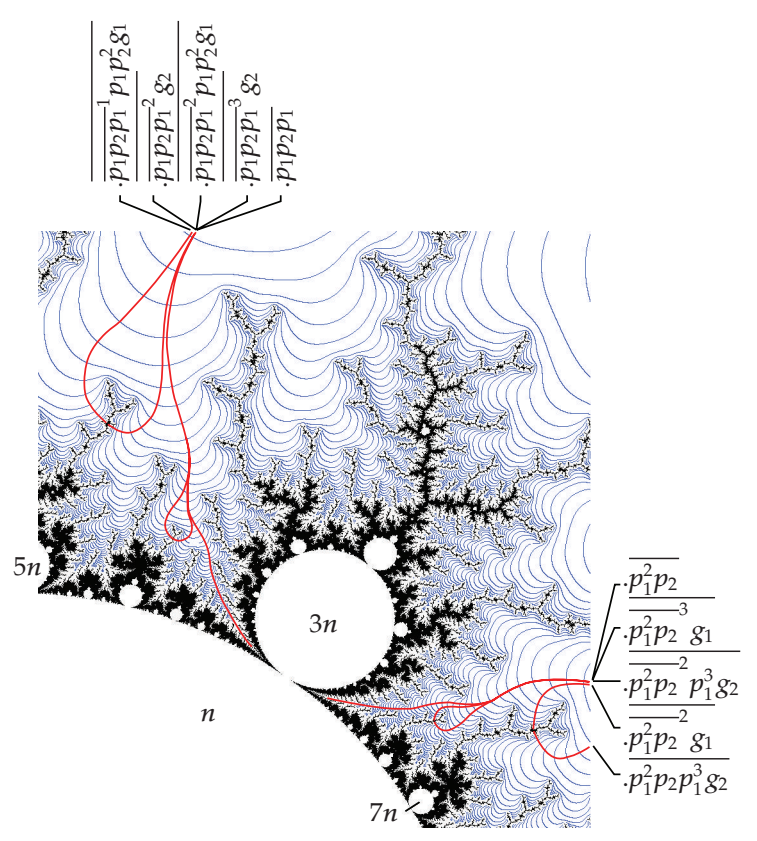

(b)

Figure 9: Canonical triple-spiral medallions $\left(\overline{\bar{p}_{1}^{2} p_{2}} p_{1}^{3} g_{2}, \overline{\bar{p}_{1}^{2} p_{2}}{ }^{i+1} g_{1}\right)$. (a) External rays of the parent and the gene. (b) Graphical location of the medallions $i=1$ and 2. (c) Detail of the medallion $i=11$ where the BMSs up to third level are pointed out. 
Table 7: Location of canonical 20.tuple-spiral medallions.

\begin{tabular}{|c|c|}
\hline$\left(\overline{\overline{p_{1}^{18} p_{2} p_{1}}}{ }^{i+1} g_{2}, \overline{{\overline{p_{1}^{18} p_{2} p_{1}}}^{i} p_{1}^{18} p_{2}^{2} g_{1}}\right)$ & Location \\
\hline$i=1$ & $-0.15450307968 \cdots+1.03099364735 \cdots i$ \\
\hline$i=2$ & $-0.154507245205 \cdots+1.030998040742 \cdots i$ \\
\hline$i=3$ & $-0.1545092214233 \cdots+1.0309995721981 \cdots i$ \\
\hline
\end{tabular}

Based on the former experimental methods, three coupling patterns are found. The canonical nonspiral medallions have a nested pairs pattern, the canonical single-spiral medallions have an adjacent pairs pattern, and the canonical (double, triple, quadruple, quintuple and 20.tuple)-spiral medallions have a 1-nested/adjacent pairs pattern. Taking into account this last thirst case, we conjecture that any canonical $n$.tuple-spiral medallion $(n \geq 2)$ has a 1-nested/adjacent pairs pattern.

\section{Acknowledgments}

This work was supported by CDTI in collaboration with Telefónica project SEGUR@ and by CDTI in collaboration with SAC project HESPERIA.

\section{References}

[1] B. B. Mandelbrot, "Fractals aspects of the iteration of $z \rightarrow \lambda z(1-z)$ for complex $\lambda, z$, " in Nonlinear Dynamics, R. H. G. Helleman, Ed., vol. 357 of Annals of New York Academy of Sciences, pp. 249-259, New York Academy of Sciences, New York, NY, USA, 1980.

[2] A. Douady and J. H. Hubbard, "Itération des polynômes quadratiques complexes," Comptes Rendus des Séances de l'Académie des Sciences. Série I, vol. 294, no. 3, pp. 123-126, 1982.

[3] A. Douady, "Algorithms for computing angles in the Mandelbrot set," in Chaotic Dynamics and Fractals, M. Barnsley and S. G. Demko, Eds., vol. 2 of Notes and Reports in Mathematics in Science and Engineering, pp. 155-168, Academic Press, Orlando, Fla, USA, 1986.

[4] A. Douady, "Julia set and the Mandelbrot set," in The Beauty of Fractals: Images of Complex Dynamical Systems, H.-O. Peitgen and P. H. Richter, Eds., pp. 161-173, Springer, Berlin, Germany, 1986.

[5] H.-O. Peitgen and P. H. Richter, The Beauty of Fractals: Images of Complex Dynamical Systems, Springer, Berlin, Germany, 1986.

[6] P. Lavaurs, "Une description combinatoire de l'involution définie par $\mathrm{M}$ sur les rationnels à dénominateur impair," Comptes Rendus des Séances de l'Académie des Sciences. Série I. Mathématique, vol. 303, no. 4, pp. 143-146, 1986.

[7] B. Branner, "The Mandelbrot set," in Chaos and Fractals: The Mathematics Behind the Computer Graphics, R. L. Devaney and L. Keen, Eds., vol. 39 of Proceedings of Symposia in Applied Mathematics, pp. 75-105, American Mathematical Society, Providence, RI, USA, 1989.

[8] L. Carleson and T. W. Gamelin, Complex Dynamics, Universitext: Tracts in Mathematics, Springer, New York, NY, USA, 1993.

[9] E. Lau and D. Schleicher, "Internal addresses in the Mandelbrot set and irreducibility of polynomials," IMS Prepint 94-19, 1994, http://www.math.sunysb.edu/cgi-bin/create_preprint_list.pl?94.

[10] D. Schleicher, "Rational parameter rays of the Mandelbrot set," IMS Preprint 97-13, 1997, http://www.math.sunysb.edu/cgi-bin/create_preprint_list.pl?97.

[11] J. Milnor, "Periodic orbits, externals rays and the Mandelbrot set: an expository account," Astérisque, no. 261, pp. 277-333, 2000.

[12] H. Bruin and D. Schleicher, "Symbolic dynamics of quadratic polynomials," Tech. Rep. 7, Institut Mittag-Leffler, Stockholm, Sweden, 2001-2002.

[13] R. L. Devaney and M. Moreno Rocha, "Geometry of the antennas in the Mandelbrot set," Fractals, vol. 10, no. 1, pp. 39-46, 2002. 
[14] J. Milnor, Dynamics in One Complex Variable, vol. 160 of Annals of Mathematics Studies, Princeton University Press, Princeton, NJ, USA, 3rd edition, 2006.

[15] W. Jung, "Mandel: software for real and complex dynamics," http://www.mndynamics.com/ indexp.html.

[16] A. Chéritat, “Dynamique holomorphe," http://www.math.univ-toulouse.fr/ cheritat/e_index.html.

[17] M. Misiurewicz, "Fragments of the Mandelbrot set," http://www.math.iupui.edu/ mmisiure/.

[18] X. Wang and Y. Sun, "The general quaternionic M-J sets on the mapping $z \leftarrow z^{\alpha}+c(\alpha \in \mathrm{N})$," Computers E Mathematics with Applications, vol. 53, no. 11, pp. 1718-1732, 2007.

[19] X. Wang and L. Gu, "Research fractal structures of generalized M-J sets using three algorithms," Fractals, vol. 16, no. 1, pp. 79-88, 2008.

[20] X. Wang, X. Zhang, Y. Sun, and F. Li, "Dynamics of the generalized M set on escape-line diagram," Applied Mathematics and Computation, vol. 206, no. 1, pp. 474-484, 2008.

[21] X. Wang and X. Zhang, "The divisor periodic point of escape-time N of the Mandelbrot set," Applied Mathematics and Computation, vol. 187, no. 2, pp. 1552-1556, 2007.

[22] X. Wang, P. Chang, and N. Gu, "Additive perturbed generalized Mandelbrot-Julia sets," Applied Mathematics and Computation, vol. 189, no. 1, pp. 754-765, 2007.

[23] X. Wang and R. Jia, "Rendering of the inside structure of the generalized M set period bulbs based on the pre-period," Fractals, vol. 16, no. 4, pp. 351-359, 2008.

[24] X. Wang, R. Jia, and Z. Zhang, "The generalized Mandelbrot set perturbed by composing noise of additive and multiplicative," Applied Mathematics and Computation, vol. 210, no. 1, pp. 107-118, 2009.

[25] X. Wang, Z. Wang, Y. Lang, and Z. Zhang, "Noise perturbed generalized Mandelbrot sets," Journal of Mathematical Analysis and Applications, vol. 347, no. 1, pp. 179-187, 2008.

[26] A. Douady, X. Buff, R. L. Devaney, and P. Sentenac, "Baby Mandelbrot sets are born in cauliflowers," in The Mandelbrot Set, Theme and Variations, T. Lei, Ed., vol. 274 of London Mathematical Society Lecture Note Series, pp. 19-36, Cambridge University Press, Cambridge, UK, 2000.

[27] R. P. Munafo, "Embedded Julia sets," http://www.mrob.com/pub/muency/embeddedjuliaset.html.

[28] M. Romera, G. Pastor, G. Alvarez, and F. Montoya, "External arguments in the multiple-spiral medallions of the Mandelbrot set," Computers and Graphics, vol. 30, no. 3, pp. 461-470, 2006.

[29] G. Pastor, M. Romera, G. Alvarez, J. Nunez, D. Arroyo, and F. Montoya, "Operating with external arguments of Douady and Hubbard," Discrete Dynamics in Nature and Society, vol. 2007, Article ID 45920, 17 pages, 2007.

[30] G. Pastor, M. Romera, G. Alvarez, et al., "Algorithm for external arguments calculation of the nodes of a shrub in the Mandelbrot set," Fractals, vol. 16, no. 2, pp. 159-168, 2008.

[31] G. Pastor, M. Romera, G. Alvarez, et al., "A general view of pseudoharmonics and pseudoantiharmonics to calculate external arguments of Douady and Hubbard," Applied Mathematics and Computation, vol. 213, no. 2, pp. 484-497, 2009.

[32] M. Romera, G. Alvarez, D. Arroyo, A. B. Orue, V. Fernandez, and G. Pastor, "Drawing and computing external rays in the multiple-spiral medallions of the Mandelbrot set," Computers and Graphics, vol. 32, no. 5, pp. 597-610, 2008.

[33] M. Romera, G. Pastor, G. Álvarez, and F. Montoya, "External arguments of Douady cauliflowers in the Mandelbrot set," Computers and Graphics, vol. 28, no. 3, pp. 437-449, 2004.

[34] G. Pastor, M. Romera, G. Álvarez, and F. Montoya, "External arguments for the chaotic bands calculation in the Mandelbrot set," Physica A, vol. 353, no. 1-4, pp. 145-158, 2005. 


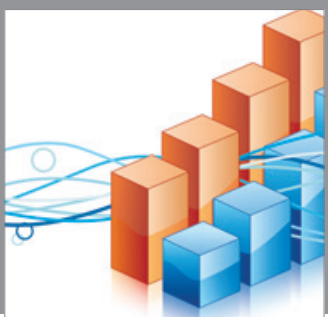

Advances in

Operations Research

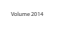

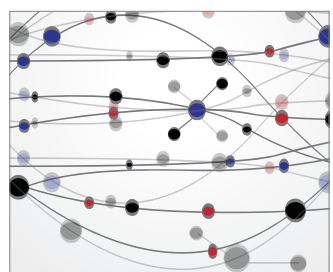

\section{The Scientific} World Journal
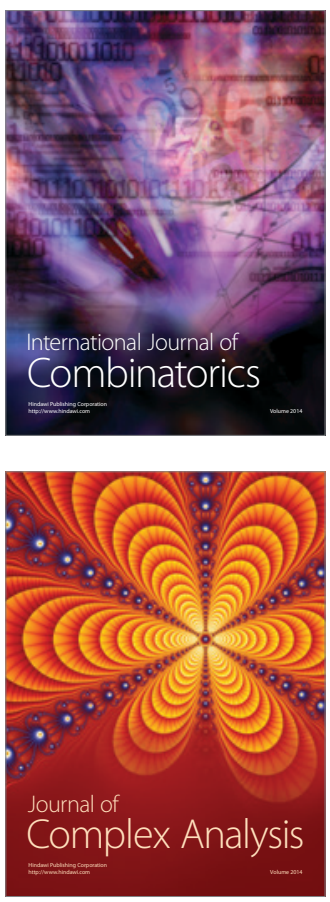

International Journal of

Mathematics and

Mathematical

Sciences
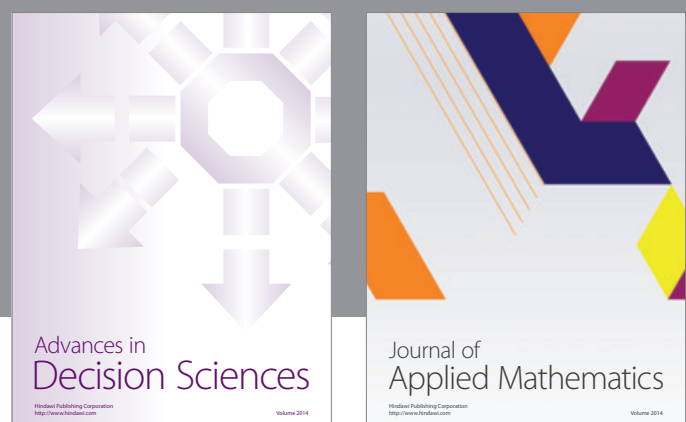

Journal of

Applied Mathematics
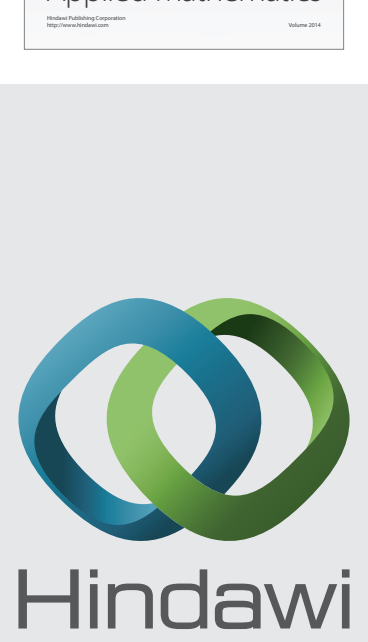

Submit your manuscripts at http://www.hindawi.com
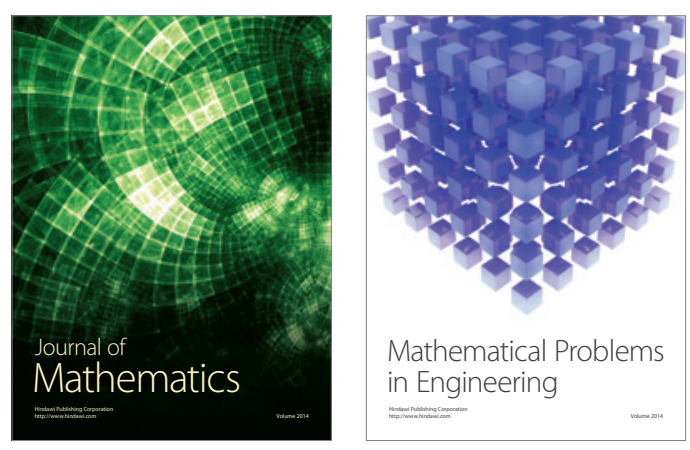

Mathematical Problems in Engineering
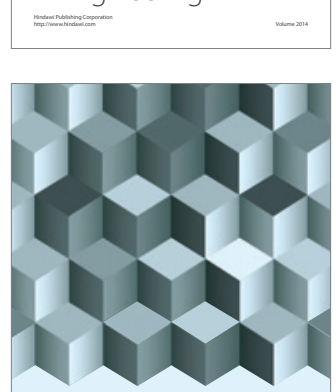

Journal of

Function Spaces
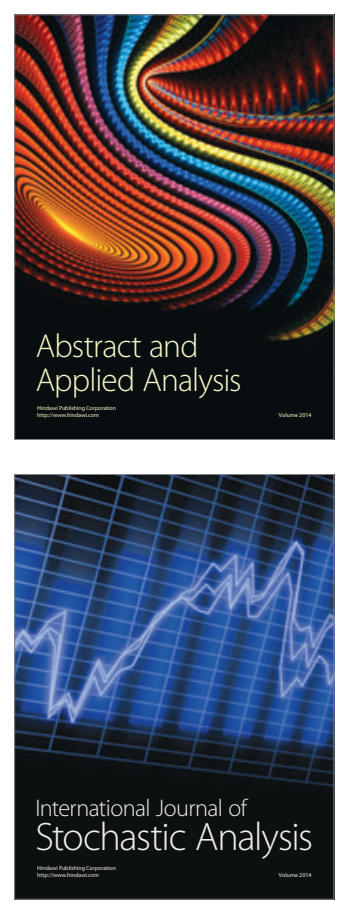

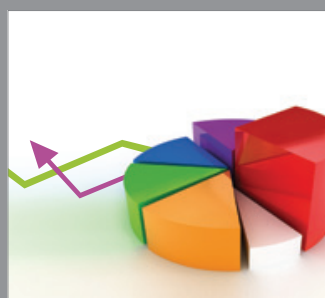

ournal of

Probability and Statistics

Promensencen
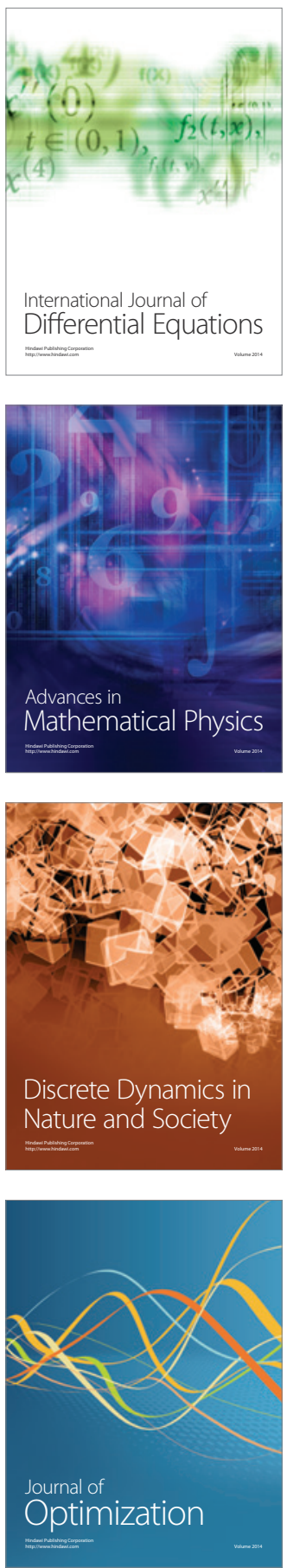